\title{
Circ_0086720 knockdown strengthens the radiosensitivity of non-small cell lung cancer via mediating the miR-375/SPIN1 axis
}

\author{
Yurong JIN, Zhenjun SU, Hui SHENG, Ke LI, Bo YANG, Shuai LI* \\ Department of Radiotherapy, Affiliated Hospital of Hebei University of Engineering, Handan, Hebei, China \\ ${ }^{*}$ Correspondence: lishuai51057@163.com
}

Received March 31, 2020 / Accepted July 1, 2020

\begin{abstract}
Radioresistance is an important cause of cancer treatment failure. Circular RNAs (circRNAs) play crucial roles in cancer development, including the radioresistance. This research aimed to determine the function and related mechanism of circ_0086720 in the radioresistance of non-small cell lung cancer (NSCLC). The expression of circ_0086720, miR-375, and Spindlin 1 (SPIN1) was measured using a quantitative real-time polymerase chain reaction (qRT-PCR). Cell survival fraction was analyzed using colony formation assay, and cell apoptosis was monitored by flow cytometry assay. The activities of caspase 3 and caspase 9 were assessed using the corresponding commercial kits. The protein levels of SPIN1 and $\gamma \mathrm{H} 2 \mathrm{AX}$ were detected by western blot. Bioinformatics analysis was performed to predict the targets of circ_0086720 and miR-375. Dual-luciferase reporter assay, RNA immunoprecipitation (RIP) assay, and RNA pull-down assay were conducted to validate the interaction between miR-375 and circ_0086720 or SPIN1. The animal model was constructed to ascertain the role of circ_0086720 in vivo. The expression of circ_0086720 and SPIN1 was increased in the radioresistant NSCLC tissues, while miR-375 expression was decreased. The circ_0086720 knockdown sensitized NSCLC cells to the radiation to further inhibit cell survival and induce cell apoptosis. Circ_0086720 targeted miR-375 and suppressed miR-375 expression, and miR-375 bound to SPIN1 to impair SPIN1 expression. miR-375 deficiency or SPIN1 overexpression could attenuate circ_0086720 knockdown-mediated radiosensitivity. The circ_0086720 knockdown also enhanced radiosensitivity to further block tumor growth in vivo. To conclude, circ_0086720 downregulation enhanced the sensitivity of NSCLC to radiation by regulating the miR-375/SPIN1 axis, contributing to the improvement of the radiotherapies in NSCLC.
\end{abstract}

Key words: circ_0086720, miR-375, SPIN1, NSCLC, radiosensitivity

Lung cancer is still the most common and prevalent malignant tumor, which is an important cause of threat to people's survival [1]. NSCLC is the main subtype of lung cancer, accounting for more than $85 \%$ of all lung cancer cases [2]. Radiotherapy is currently the main non-surgical treatment for NSCLC and destroys the DNA of tumor cells, leading to cell death [3]. However, the efficacy of radiotherapy is limited because of acquired radioresistance during the treatment, leading to a poor prognosis [4-6]. Accumulating evidence shows that biomarkers, such as non-coding RNAs (ncRNAs), play a substantial role in the prediction of radioresistance [7]. Therefore, exploring the mechanism of radiotherapy resistance and identifying new therapeutic targets will help improve the effectiveness of radiotherapy.

Circular RNAs (circRNAs), a class of ncRNAs, generate from precursor mRNAs by "back-splicing" [8]. CircRNAs are different from linear mRNAs due to their unique closed-loop structure without 5' end cap and a 3' end tail [9]. Therefore, they have higher stability compared to linear RNAs and are more suitable as biomarkers in different human diseases [10]. Recently, circRNAs were reported to be dysregulated in radioresistant and radiosensitive tumor tissues and play functions in radioresistance in various cancers [11, 12], such as circ_0001313 in colon cancer [13], circ_100367 in esophageal squamous cell carcinoma [14], and circ_000543 in nasopharyngeal carcinoma [15]. In NSCLC, numerous circRNAs were documented to promote or suppress cancer progression $[16,17]$. However, related research of circRNAs in radioresistance in NSCLC is still lacking. A previous study provided several differently expressed circRNAs between radioresistant and radiosensitive esophageal cancer cells, and circ_0086720 (circRNA_104763), deriving from UBAP2, was one of the upregulated circRNAs in radioresistant esophageal cancer (EC) cells compared with that in radiosensitive EC cells [11]. It is worth exploring whether circ_0086720 has abnormal expression and special function in radioresistant NSCLC. microRNAs (miRNAs) are a cluster of short and conservative ncRNAs and play important roles in tumor initia- 
tion, development, and inhibition [18]. Studies indicated that miRNAs were associated with radiotherapy and novel targeted therapy [19]. miR-375 was demonstrated to be downregulated in NSCLC and act as a tumor suppressor to block NSCLC progression [20]. However, its role in radioresistance of NSCLC is lacking. Spindlin 1 (SPIN1) is one membrane of the SPIN/SSTY family and closely associated with spindle organization and chromosomal stability [21]. Recently, SPIN1 acted as an oncogene to promote the development of various cancers and was regarded as an ideal biomarker in cancer treatment [22]. Besides, SPIN1 overexpression was always linked to enhanced chemoresistance in cancers [23]. However, little is known of SPIN1 action in NSCLC radioresistance and relevant mechanism.

At present, we distinguished the expression of circ_0086720 in radioresistant and radiosensitive NSCLC tissues. Lossfunction experiments were performed to investigate the function of circ_0086720 in radioresistance in NSCLC cells and mice models. In addition, the interaction of miR-375 and circ_0086720 or SPIN1 was validated. This paper intended to provide a potential mechanism of circ_0086720 in understanding NSCLC development with radioresistance.

\section{Patients and methods}

Tissues. Tumor specimens and adjacent normal tissue specimens were collected from 52 NSCLC patients recruited from the Affiliated Hospital of the Hebei University of Engineering. All patients were pathologically diagnosed with NSCLC and did not receive any chemotherapy or radiotherapy before. After receiving radiotherapy, the radiotherapy response was evaluated to determine the radiotherapy resistance and sensitivity. That was, the tumor volume reduction rate less than $30 \%$ was considered to be radioresistant, and tumor volume reduction rate $>30 \%$ was considered to be radiosensitive or partially sensitive. All specimens were collected during tumor resection, immediately placed in liquid nitrogen, and stored at $-80^{\circ} \mathrm{C}$. All patients signed informed consent before surgery. This study was conducted with the approval of the Ethics Committee of the Affiliated Hospital of the Hebei University of Engineering.

Cell lines. NSCLC cells (A549 and H1299) and normal bronchial epitheliums (BEAS-2B) purchased from Procell Co., Ltd. (Wuhan, China) were cultured in 90\% Roswell Park Memorial Institute 1640 (RPMI 1640; Procell Co., Ltd.) medium containing $10 \%$ fetal bovine serum (FBS, Procell Co., Ltd.) and bronchial epithelial cell growth medium (Procell Co., Ltd.) containing 10\% FBS, respectively. All cells were maintained in the corresponding medium and placed at $37^{\circ} \mathrm{C}$ conditions containing $5 \% \mathrm{CO}_{2}$.

Cell transfection. Small interference RNA targeting circ_0086720 (si-circ\#1 and si-circ\#2), short hairpin RNA lentiviral vector targeting circ_0086720 (sh-circ) and their negative control (si-NC or sh-NC) were assembled by GeneCopoeia (Guangzhou, China). miR-375 mimics
(miR-375), inhibitors (anti-miR-375) and their negative controls (miR-NC and anti-NC) were purchased from Ribobio (Guangzhou, China), SPIN1 overexpression vector (oe-SPIN1), and its control (vector) were constructed using pcDNA3.1 overexpression vector by Ribobio. For cell transfection, A549 and H1299 cells $\left(1 \times 10^{6}\right)$ were introduced with the above transfection using Lipofectamine 3000 Reagent (Invitrogen, Carlsbad, CA, USA).

Radiation treatment. Cells seeded into 96-well plates were exposed to 6-MV X-ray irradiation at a dose rate of 2 Gy/min by a linear accelerator (Varian Medical Systems, Palo Alto, CA, USA), with a total dose of 0, 2, 4, or 8 Gy. After 24 $\mathrm{h}$, cells were collected for the following analyses.

Quantitative real-time polymerase chain reaction (qRT-PCR). Total RNA was obtained using TRIzol reagent (Invitrogen) and subjected to reverse transcription using the First-Strand Synthesis SuperMix for qRT-PCR (Invitrogen) for circ_0086720 and SPIN1 or using the TaqMan MicroRNA Reverse Transcription Kit (Applied Biosystems, Foster City, CA, USA) for miR-375. Subsequently, qRT-PCR was carried out using the SYBR Green PCR master mix (Applied Biosystems) in line with its protocol. Glyceraldehyde-3-phosphate dehydrogenase (GAPDH) served as an endogenous control to normalize circ_0086720 and SPIN1 expression, and U6 served as an endogenous control for miR-375. The relative expression was assessed using the $2^{-\Delta \Delta C \mathrm{Ct}}$ method. The primer sequences were: circ_0086720, F: 5'-AAGCCTCAGAAGCCAACTCC-3' and R: 5'-GTCACTTCCATAAGCAGGCTCT-3', miR-375, F: 5'-TGCGGTTTGTTCGTTCGGCT-3' and R: 5'-CAGTGCAGGGTCCGAGGT-3', SPIN1, F: 5'-CAGAGCTGATGCAGGCCAT-3' and R: 5'-ACTGGGTAACAGGGCCATTG-3'. GAPDH, F: 5'- TGCACCACCAACTGCTTA-3' and R: 5'-GGATGCAGGGATGATGTTC-3', U6, F: 5'-GCTTCGGCAGCACATATACTAAAAT-3' and R: 5'- CGCTTCACGAATTTGCGTGTCAT-3'.

Colony formation assay. Cell survival fraction was determined by a colony-forming assay. Briefly, $1 \times 10^{5}$ cells with different transfection were plated into a 6-well plate and subjected to different doses of radiation $(0,2,4$, and $8 \mathrm{~Gy})$. The plate was then placed into a $37^{\circ} \mathrm{C}$ incubator for $12 \mathrm{~d}$ for colony formation. After that, the colonies were washed with phosphate-buffered saline (PBS), fixed with formaldehyde, and stained with crystal violet. The colony phenotype was observed under a microscope. A colony containing $>50$ cells was served as one colony. The survival fractions (SFs) were calculated following the equation: the number of colonies/ (number of plated cells $\times$ plating efficiency) [24].

Flow cytometry assay. Cells with different transfection were exposed to radiation. For apoptosis assay, cells were harvested at $24 \mathrm{~h}$ post-radiation and stained with Annexin V-fluorescein isothiocyanate (FITC) and propidium iodide (PI) using the Annexin V-FITC Apoptosis Detection Kit (Beyotime, Shanghai, China) according to the instructions. Flow cytometry assay was performed using a FACScan flow cytometer (BD Bioscience; San Jose, CA, USA). 
Caspase 3 and caspase 9 activity detection. Cells with different transfection and exposed to radiation were collected and washed with PBS. Then, the activities of caspase 3 and caspase 9 were detected using the Caspase 3 activity assay kit (Beyotime) and Caspase 9 activity assay kit (Beyotime), respectively. All procedures were conducted in agreement with the instructions.

Western blot. Western blot was performed to detect the expression of $\gamma \mathrm{H} 2 \mathrm{AX}$ (a marker of DNA damage and repair) and SPIN1. The isolated proteins were separated and transferred onto polyvinylidene fluoride (PVDF) membranes. After incubation with block buffer, the membranes were exposed to the primary antibodies: anti- $\gamma \mathrm{H} 2 \mathrm{AX}$ (ab2893; Abcam Cambridge, MA, USA), anti-SPIN1 (ab118784; Abcam), and anti-GAPDH (ab9485; Abcam). After that, the membranes were exposed to goat anti-rabbit secondary antibody (ab205718; Abcam). Finally, the protein blots were emerged using the enhanced chemiluminescence plus (Beyotime).

Bioinformatics analysis. The target miRNAs were predicted using the Circinteractome (https://circinteractome.nia.nih.gov/). The target mRNAs of miR-375 were predicted using the starBase (http://starbase.sysu.edu.cn/).

Dual-luciferase reporter assay. Wild-type and mutant nucleotide sequences (mutated at the binding site) of circ_0086720 and SPIN1 3'UTR were inserted into the pmirGLO vector (Promega, Madison, WI, USA) to generate luciferase reporter plasmids, naming as WT-circ_0086720, MUT-circ_0086720, WT-SPIN1 3'UTR, and MUT-SPIN1 3'UTR, respectively. A549 and H1299 cells plated into 96-well plates were transfected with miR-375 (50 nM) or a miR-NC and WT-circ_0086720, MUT-circ_0086720, WT-SPIN1 3'UTR, or MUT-SPIN1 3'UTR, respectively. Cells after transfection were incubated for $48 \mathrm{~h}$ and collected for luciferase activity detection using the dual-luciferase reporter assay (Promega).

RNA immunoprecipitation (RIP) assay. RIP assay was conducted according to the experimental procedures from the EZ-Magna RIP kit (Millipore, Billerica, MA, USA). Antibodies against Argonaute 2 (Ago2; Abcam) and Immunoglobulin G (IgG; Abcam) were utilized for RIP. The immunoprecipitated RNAs were isolated, and qRT-PCR was performed in line with the abovementioned methods to detect the expression of circ_0086720 and miR-375.

RNA pull-down assay. miR-375 and miR-NC were labeled using biotin by Ribobio, terming as bio-miR-375 and bio-miR-NC. A549 and H1599 cells were transfected with bio-miR-375 or bio-miR-NC and lysed in Lysis Buffer from the Pierce Magnetic RNA-Protein Pull-Down Kit (Thermo Fisher Scientific, Waltham, MA, USA). Then the lysates were reacted with streptavidin magnetic beads at $4{ }^{\circ} \mathrm{C}$ overnight. Finally, the bound RNAs were eluted and isolated for qRT-PCR assay to detect the expression of circ_0086720.

Tumor growth in vivo. Animal experimental procedures obtained the authorization of the Animal Care and Use Committee of the Affiliated Hospital of the Hebei University of Engineering. BALB/c nude mice (male, aged 4-6 weeks, $\mathrm{n}=15$ ) were purchased from Vital River Laboratories (Beijing, China) and housed in standard conditions. A549 cells transfected with sh-circ or sh-NC were cultured in culture medium to $70-80 \%$ density. The nude mice were randomly separated into three groups ( $n=5 /$ group) and injected with a density of $2 \times 10^{6}$ A 549 cells (with sh-NC transfection for two groups and with sh-circ for one group) in the left flank. Tumor growth was monitored frequently until the tumor volume reached an average volume of $300 \mathrm{~mm}^{3}$. Then, a group of mice with sh-NC transfection and a group of mice with sh-circ transfection were irradiated with a dose of 4 Gy once every day for 5 days. Tumor volume was measured once a week after radiation. At 28th day post-radiation, all mice were sacrificed by cervical dislocation and used for subsequent experiments. The tumor volume was calculated according to the formula: $0.5 \times$ length $\times$ width $^{2}$.

Statistics analysis. Data were obtained from three independent biological experiments and processed using GraphPad Prism 7.0 software (GraphPad Prism, La Jolla, CA, USA). The results of the data were shown as the mean \pm standard deviation. Differences between the two groups were analyzed by Student's t-test. A one-way analysis of variance plus Tukey post hoc test was conducted to evaluate the differences among multiple groups. The expression correlations between miR-375 and circ_0086720 or SPIN1 in NSCLC tissues were assessed using Spearman's correlation analysis. A p-value $<0.05$ represented statistically significant difference.

\section{Results}

Circ_0086720 was highly expressed in radioresistant NSCLC tissues, while miR-375 was weakly expressed. Circ_0086720 was generated from UBAP2 mRNA by backsplicing, and the schematic was depicted to illustrate the generation of circ_0086720 (Figure 1A). The expression of circ_0086720 and miR-375 was detected in clinical tissues, and the data manifested that the expression of circ_0086720 was strikingly increased in NSCLC tissues compared with normal tissues, while the expression of miR-375 was notably declined in NSCLC tissues compared with normal tissues (Figures 1B, 1C). Through the Spearman's correlation analysis, miR-375 expression was negatively correlated with circ_0086720 expression in NSCLC tissues (Figure 1D). Besides, higher expression of circ_0086720 was observed in radioresistant NSCLC tissues compared to radiosensitive NSCLC tissues, while the expression of miR-375 in radioresistant NSCLC tissues was lower than that in radiosensitive NSCLC tissues (Figures 1E, 1F). These data suggested that circ_0086720 and miR-375 were dysregulated in NSCLC tissues, partially in radioresistant NSCLC tissues.

Circ_0086720 knockdown enhanced radiosensitivity in NSCLC cells to inhibit colony formation and induce apoptosis. The expression of circ_0086720 was 
A

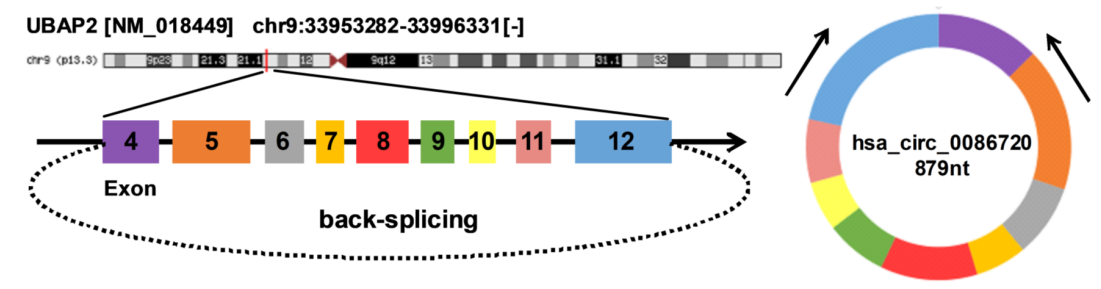

B

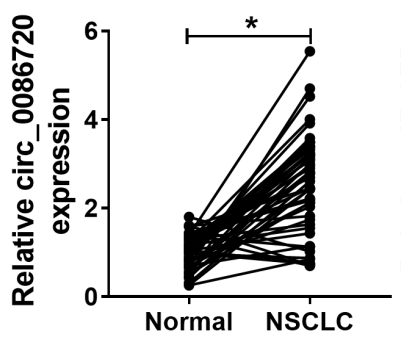

E

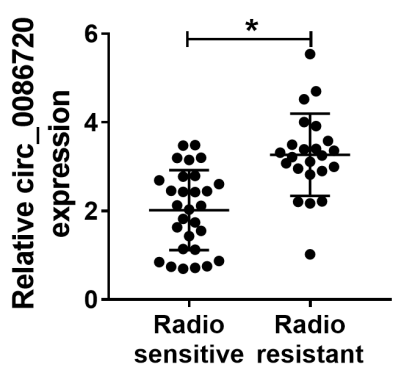

C

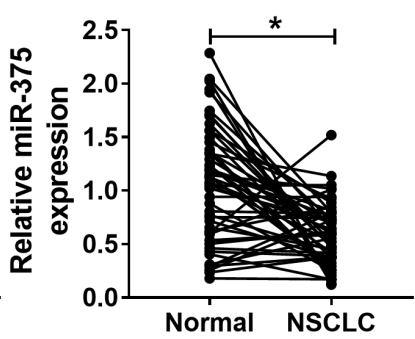

$\mathbf{F}$

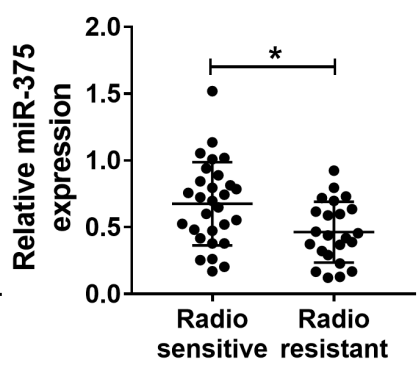

D

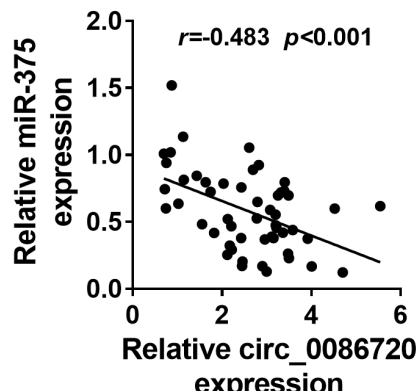

expression

Figure 1. Circ_0086720 was upregulated in NSCLC tissues, while miR-375 was downregulated in NSCLC tissues. A) Schematic illustrated the generation of circ_0086720. B, C) The expression of circ_0086720 and miR-375 in NSCLC tissues $(\mathbf{n}=52)$ and normal tissues ( $\mathrm{n}=52$ ) was detected by qRT-PCR. D) The correlation between miR-375 expression and circ_0086720 expression in NSCLC tissues was analyzed by Spearman's correlation analysis. E, F) The expression of circ_0086720 and miR-375 in radiosensitive $(n=29)$ or radioresistant $(n=23)$ NSCLC tissues was detected by $q R T-P C R$. ${ }^{*} \mathbf{p}<0.05$

also markedly elevated in A549 and H1299 cells compared with that in BEAS-2B cells (Figure 2A). We used siRNA to weaken circ_0086720 expression, and the efficiency of interference was detected by qRT-PCR. The data showed that circ_0086720 was significantly downregulated in A549 and H1299 cells after transfection of si-circ\#1 and si-circ\#2 (Figure 2B). The expression of circ_0086720 decreased more in cells transfected with si-circ\#1 than with si-circ\#2, thus cells transfected with si-circ\#1 were used for the following experiments. A549 and $\mathrm{H} 1299$ cells were exposed to radiation $(0,2,4$, and $8 \mathrm{~Gy})$, and the colony formation assay presented that the survival fraction of cells transfected with si-circ\#1 or si-NC was both declined in a dose-independent manner. Besides, the survival fraction was significantly lower in cells transfected with si-circ\#1 compared to si-NC (Figures 2C, 2D). Compared to no radiation, $4 \mathrm{~Gy}$ radiation significantly induced cell apoptosis, and the apoptosis rate was remarkably promoted in cells transfected with si-circ\#1 compared to si-NC in A549 and H1299 cells exposed to radiation
(4Gy) (Figure 2E). Additionally, the activities of caspase 3 and caspase 9 were induced in 4 Gy radiation-treated cells, and si-circ\#1 transfection further motivated their activities compared with si-NC transfection (Figures 2F, 2G). The expression of $\gamma \mathrm{H} 2 \mathrm{AX}$ was also promoted in 4 Gy radiationtreated cells, and si-circ\#1 transfection further strengthened its expression (Figure $2 \mathrm{H}$ ). These data suggested that circ_0086720 knockdown could enhance radiosensitivity of NSCLC cells, inducing cell apoptosis.

miR-375 was a target of circ_0086720. Subsequently, we performed experiments to validate the interaction between circ_0086720 and miR-375. The expression of miR-375 was significantly declined in A549 and H1299 cells compared with that in BEAS-2B cells (Figure 3A). After obtaining miR-375 from the bioinformatics tool (Circinteractome), the wildtype sequence of circ_0086720 was mutated at the miR-375 binding site to generate a mutant sequence of circ_0086720, and two sequences were inserted into the downstream of pmiRGLO vector to conduct dual-luciferase reporter assay 
A
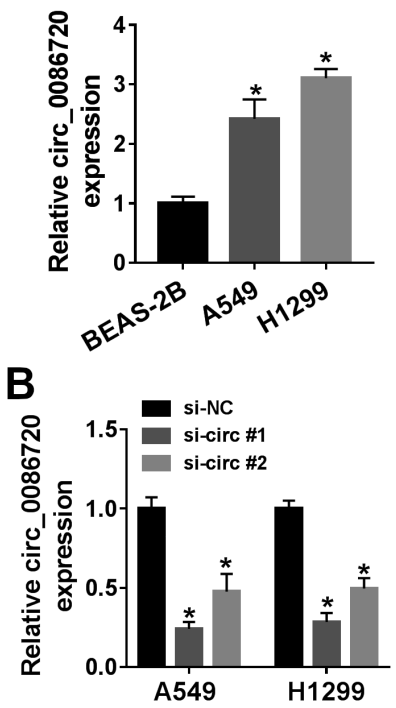

E

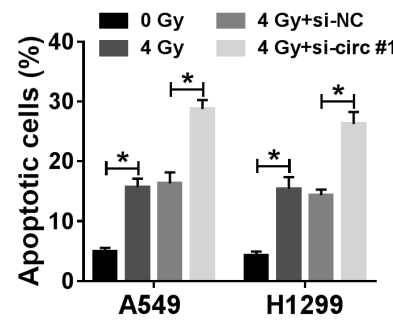

G

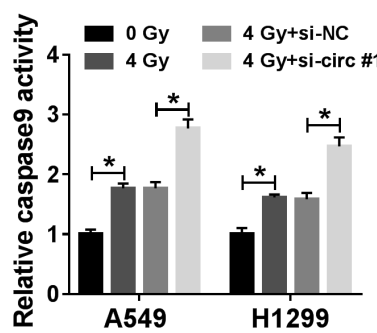

C
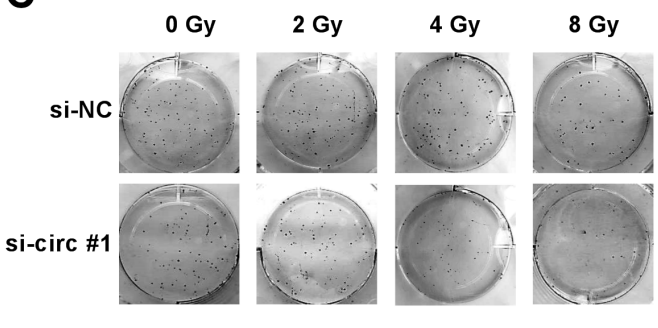

D
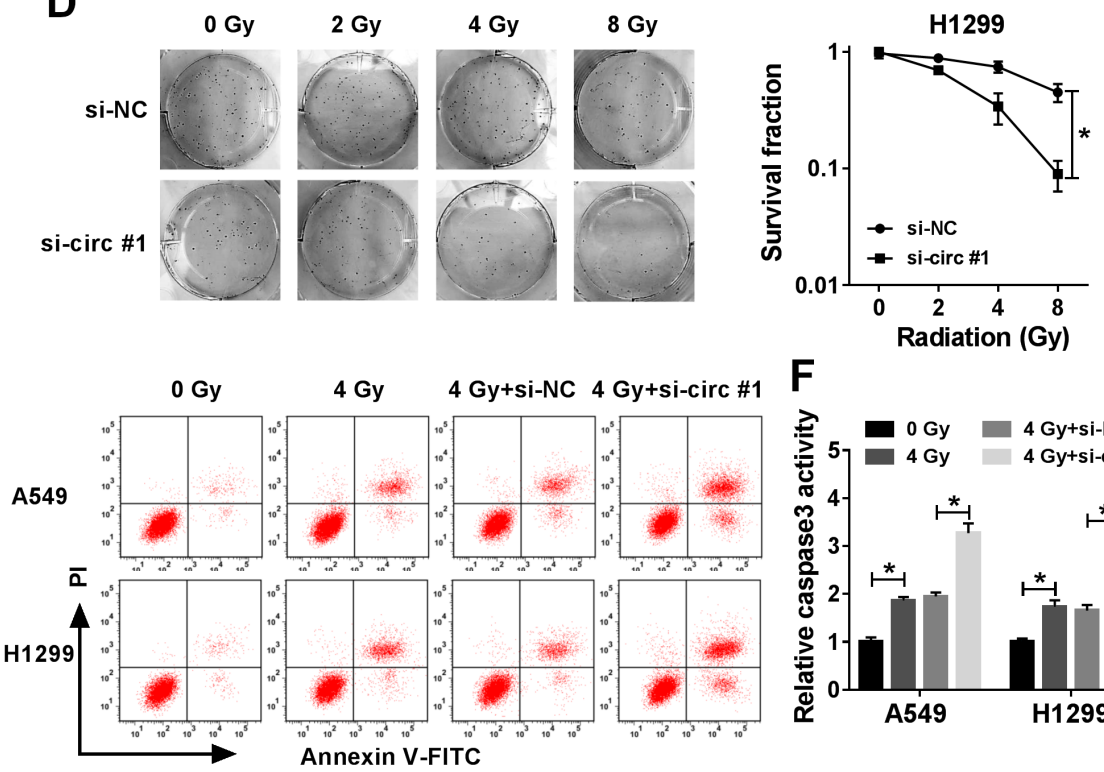

$F$

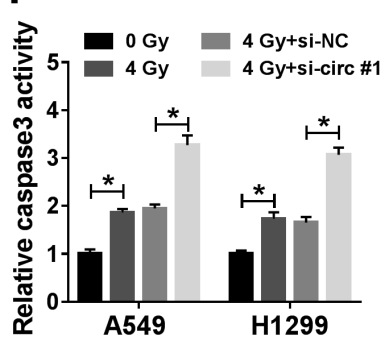

$\mathrm{H}$

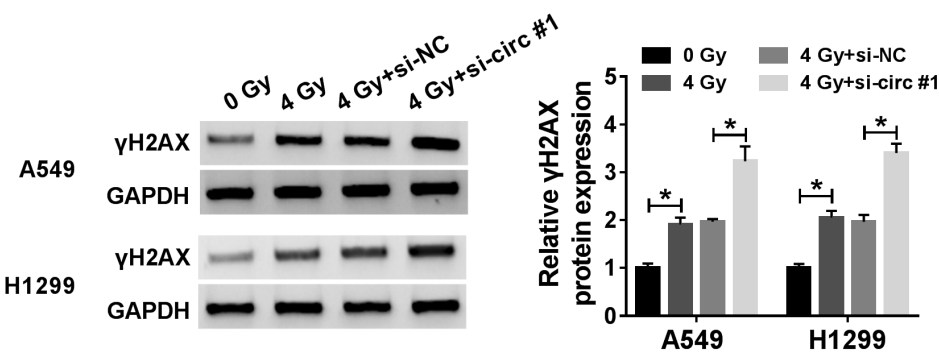

Figure 2. Circ_0086720 knockdown strengthened radiosensitivity in vitro. A) The expression of circ_0086720 in BEAS-2B, A549, and H1299 cells was measured by qRT-PCR. B) The efficiency of circ_0086720 knockdown was checked by qRT-PCR. C, D) The effect of circ_0086720 on colony formation in radiation-treated A549 and $\mathrm{H} 1299$ cells was assessed by colony formation assay. E) Cell apoptosis was detected using a flow cytometry assay. F, G) The activities of caspase 3 and caspase 9 were monitored using the corresponding kits. $\mathrm{H}$ ) The expression of $\gamma \mathrm{H} 2 \mathrm{AX}$ was measured by western blot. ${ }^{*} \mathrm{p}<0.05$

(Figure 3B). The introduction of miR-375 notably reinforced the expression of miR-375 compared to miR-NC (Figure 3C), and miR-375 reintroduction significantly diminished the luciferase activity in A549 and H1299 cells transfected with WT-circ_0086720 but not MUT-circ_0086720 compared with the miR-NC introduction (Figure 3D). In addition, both circ_0086720 and miR-375 were abundantly detected in the Ago2 RIP group compared to the IgG RIP group (Figure 3E). Moreover, RNA pull-down assay uncovered that circ_0086720 was substantially enriched by bio-miR-375 in A549 and H1299 cells but not bio-miR-NC (Figure 3F). Additionally, miR-375 expression was dramatically reinforced in cells after circ_0086720 knockdown (Figure 3G). All data disclosed that miR-375 was targeted by circ_0086720.

miR-375 deficiency reversed circ_0086720 knockdownmediated radiosensitivity in A549 and $\mathrm{H} 1299$ cells. The expression of miR-375 was prominently lessened in A549 and H1299 cells transfected with anti-miR-375 compared 
A

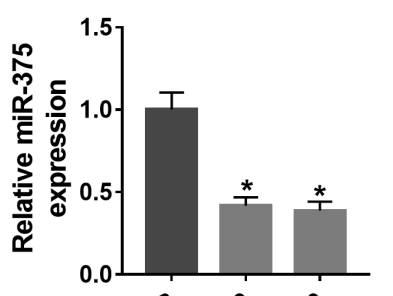

D

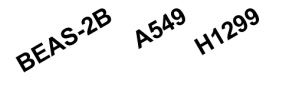

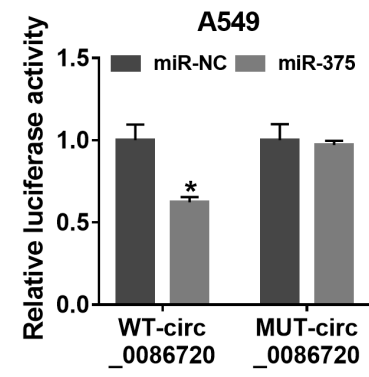

$\mathbf{F}$

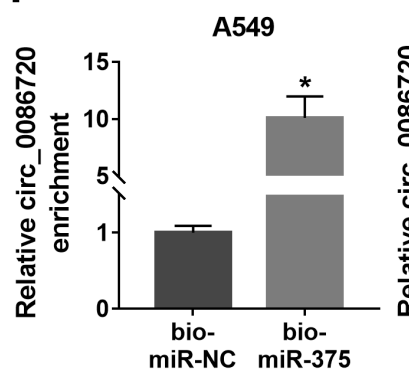

B

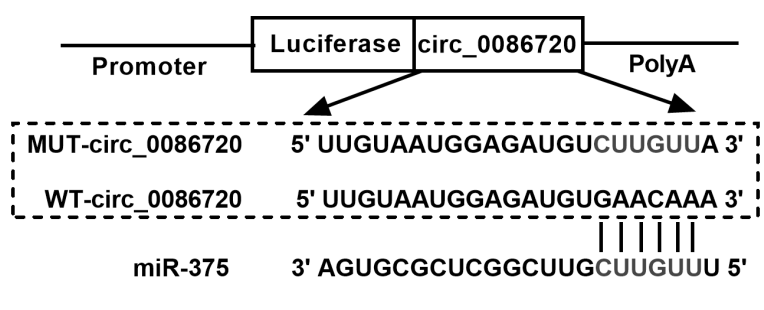

E

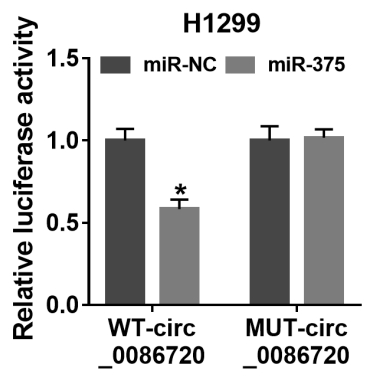

H1299

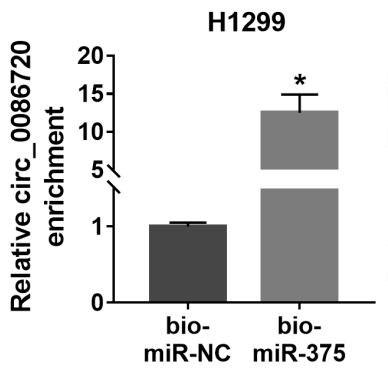

\section{G}

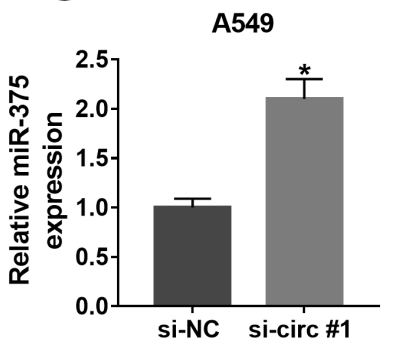

C

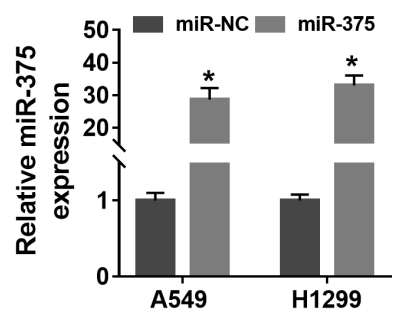

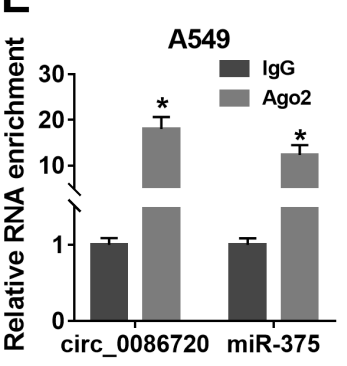
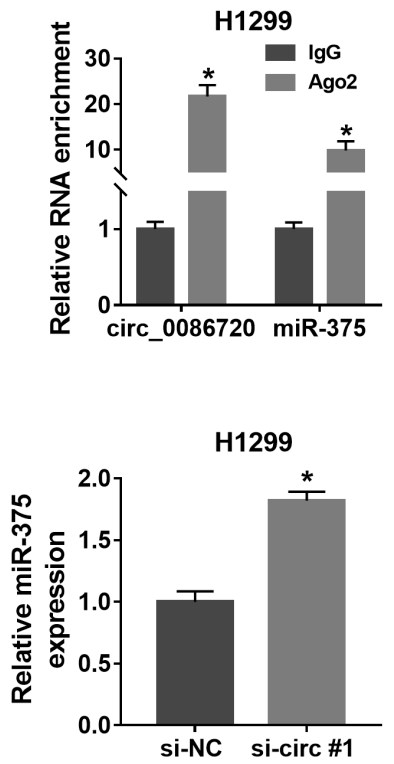

Figure 3. miR-375 was a target of circ_0086720. A) The expression of miR-375 in BEAS-2B, A549, and H1299 cells was detected by qRT-PCR. B) The binding site between circ_0086720 and miR-375 was obtained from the bioinformatics tools (Circinteractome). C) The expression of miR-375 in A549 and H1299 cells transfected with miR-375 or miR-NC was detected by qRT-PCR. D) Dual-luciferase reporter assay, E) RIP assay and F) RNA pull-down assay was performed to validate the interaction between circ_0086720 and miR-375. G) The expression of miR-375 in cells transfected with si-circ\# 1 or si-NC was detected by qRT-PCR. * $\mathrm{p}<0.05$

to anti-NC (Figure 4A). Then, A549 and H1299 cells were transfected with si-circ\#1+anti-miR-375 or si-circ\#1+antiNC to ascertain the interaction between circ_0086720 and miR-375. In A549 and H1299 cells exposed to radiation (0, 2 , 4 , and $8 \mathrm{~Gy}$ ), the survival fraction was substantially recovered by si-circ\#1+anti-miR-375 transfection compared with si-circ\#1+anti-NC transfection (Figure 4B). Besides, si-circ\#1 transfection strengthened the effects of radiation to further promote cell apoptosis, however, anti-miR-375 reintroduction weakened these effects to diminish the apoptosis rate (Figure 4C). Caspase 3 and caspase 9 activities stimulated by radiation treatment were further promoted by circ_0086720 knockdown but partly inhibited by simultaneous miR-375 deficiency (Figure 4D, 4E). The expression of $\gamma \mathrm{H} 2 \mathrm{AX}$ also induced by radiation treatment was further activated by circ_0086720 knockdown but partly decreased by simultaneous miR-375 deficiency (Figure 4F). These data suggested that circ_0086720 knockdown enhanced radiosensitivity partly by enriching the expression of miR-375.

miR-375 bound to SPIN1, which was upregulated in radioresistant NSCLC tissues. We further identified the potential target mRNAs of miR-375 by starBase, and SPIN1 was one of the targets of miR-375. The wild-type sequence of SPIN1 3'UTR (harboring miR-375 binding site) and the mutant sequence of SPIN1 3'UTR (harboring mutated miR-375 binding site) were inserted into the pmiRGLO vector (Figure 5A), and the data from dual-luciferase reporter assay showed that $\mathrm{miR}-375$ reintroduction prominently reduced the luciferase activity in A549 and H1299 cell with WT-SPIN1 3'UTR transfection but not MUT-SPIN1 3'UTR transfection (Figures 5B, 5C). Besides, SPIN1 expression was weakened in A549 and H1299 cells after miR-375 enrichment but significantly promoted in cells after miR-375 deficiency (Figures 5D, 5E). The expression of SPIN1 was 
aberrantly upregulated in A549 and H1299 cells compared with BEAS-2B cells (Figure 5F), and SPIN1 expression was also elevated in NSCLC tissues $(n=52)$ compared to normal tissues $(n=52)$ (Figure 5G, 5H). Moreover, the expression of SPIN1 was significantly higher in radioresistant NSCLC tissues $(n=23)$ compared to radiosensitive NSCLC tissues $(n=29)$ (Figure 5I, 5J). Furthermore, miR-375 expression was negatively correlated with SPIN1 expression in NSCLC tissues (Figure 5K). These data manifested that SPIN1 was a target of miR-375, and SPIN1 was highly expressed in NSCLC tissues, particularly in radioresistant NSCLC tissues.
SPIN1 overexpression reversed circ_0086720 knockdown-mediated radiosensitivity in A549 and H1299 cells. The expression of SPIN1 was impaired in A549 and H1299 cells transfected with si-circ\# $1+$ anti-NC compared to si-NC+anti-NC but substantially restored in cells transfected with si-circ\#1+anti-miR-375 compared to si-circ\#1+antiNC (Figure 6A), suggesting that circ_0086720 knockdown diminished the expression of SPIN1 by regulating miR-375. Then, we increased the expression of SPIN1 to perform rescue experiments. The expression of SPIN1 was significantly reinforced in cells transfected with oe-SPIN1
A

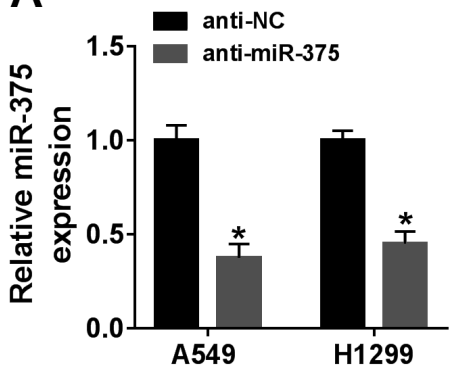

C

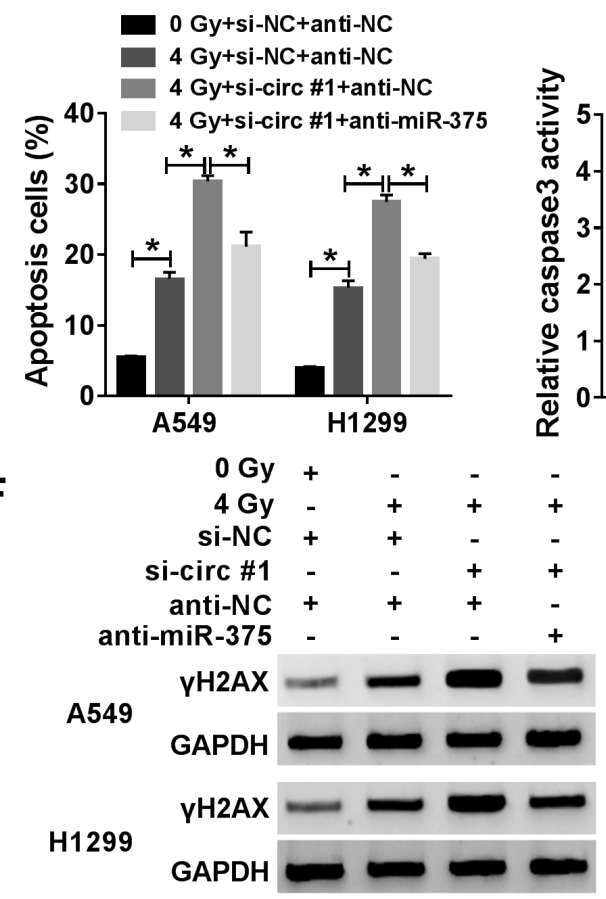

B

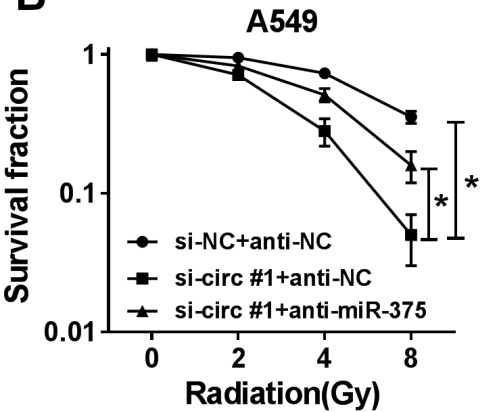

D

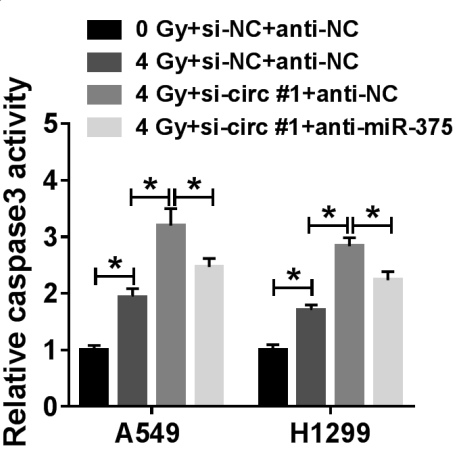

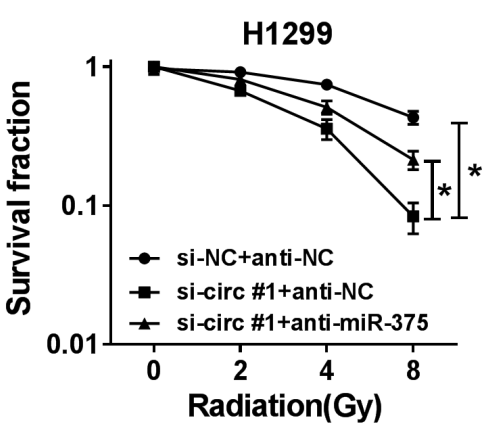

$\mathbf{E}$

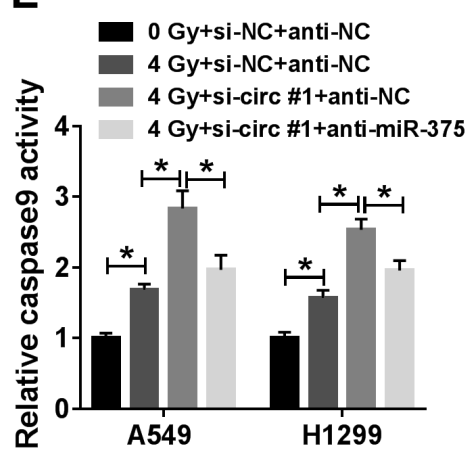

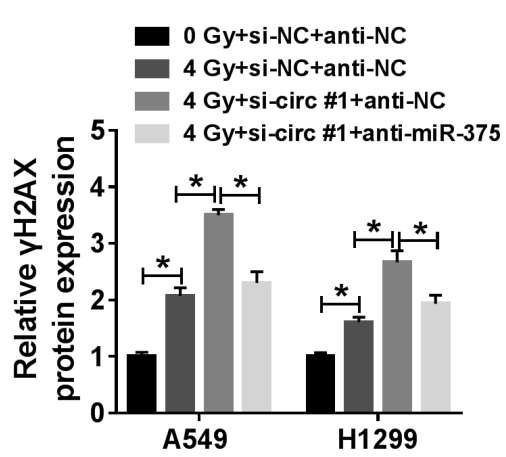

Figure 4. miR-375 deficiency attenuated the enhancement effect of circ_0086720 knockdown on radiosensitivity. A) The expression of miR-375 in A549 and $\mathrm{H} 1299$ cells transfected with anti-miR-375 or anti-NC was detected by qRT-PCR. B) Colony formation assay determined colony formation ability in different doses of radiation-treated A549 and H1299 cells with si-circ\#1+anti-miR-375 or si-circ\#1+anti-NC transfection. In A549 and H1299 cells exposed to 0 or 4 Gy radiation and transfected with si-NC+anti-NC, si-circ\# 1+anti-miR-375 or si-circ\# 1+anti-NC, C) cell apoptosis was assessed by flow cytometry assay. D, E) The activities of caspase 3 and caspase 9 were measured using the corresponding kits. F) The expression of $\gamma \mathrm{H} 2 \mathrm{AX}$ was examined using western blot. ${ }^{*} \mathbf{p}<0.05$ 
A

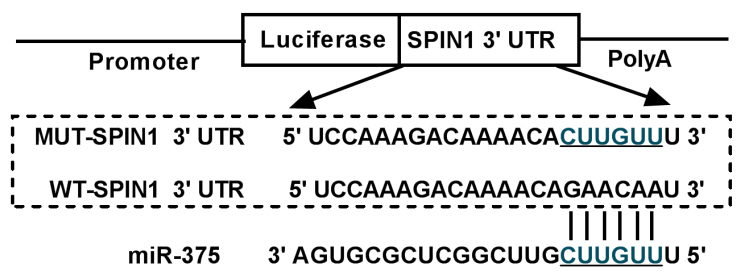

D

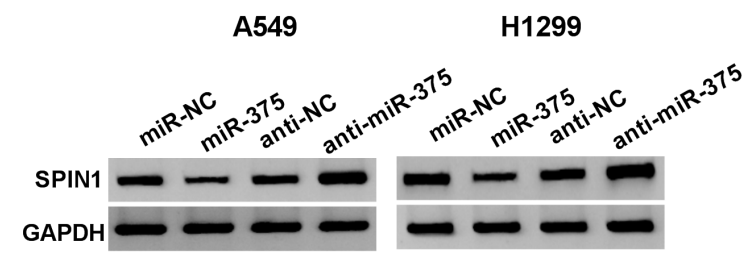

$F$
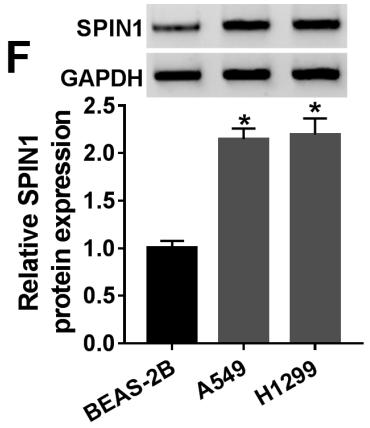

I

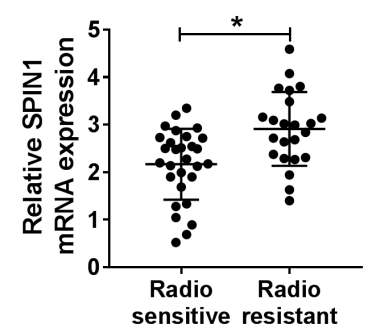

G

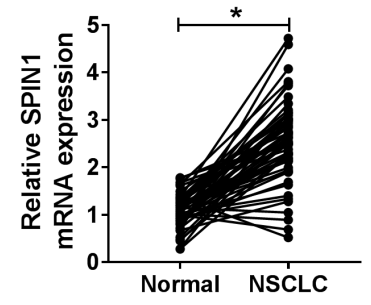

$J$

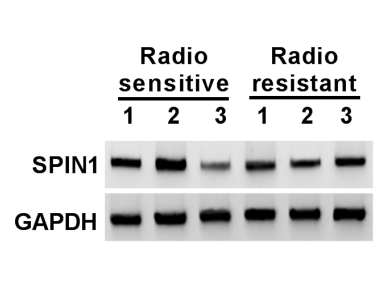

B

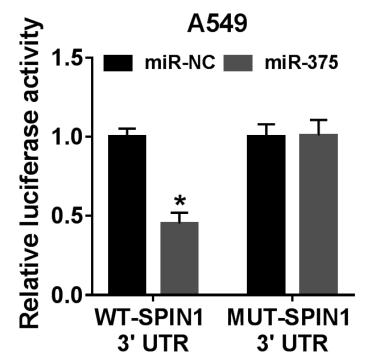

E

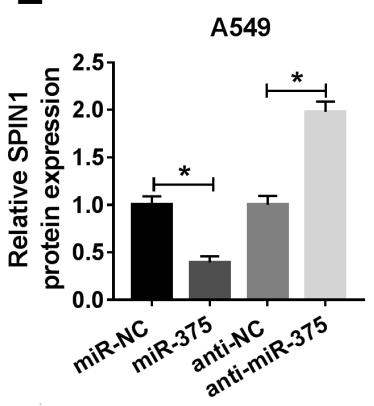

C

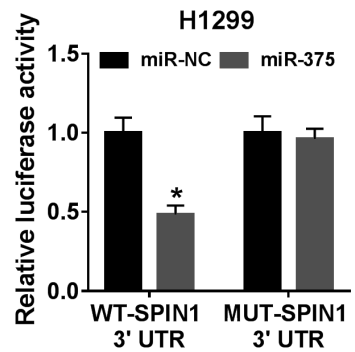

H1299
H

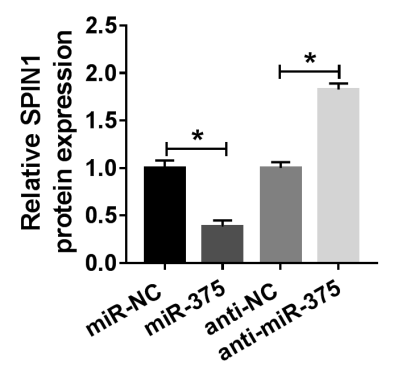

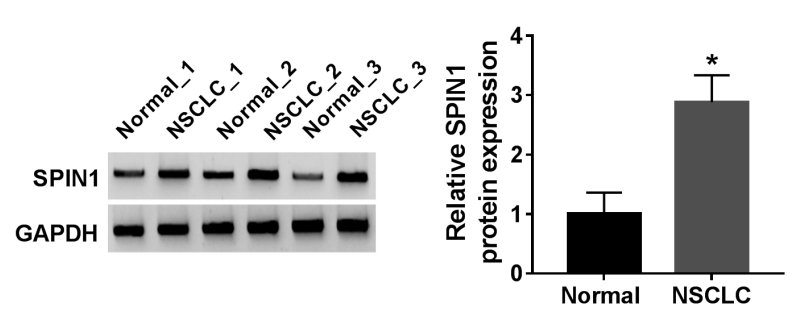
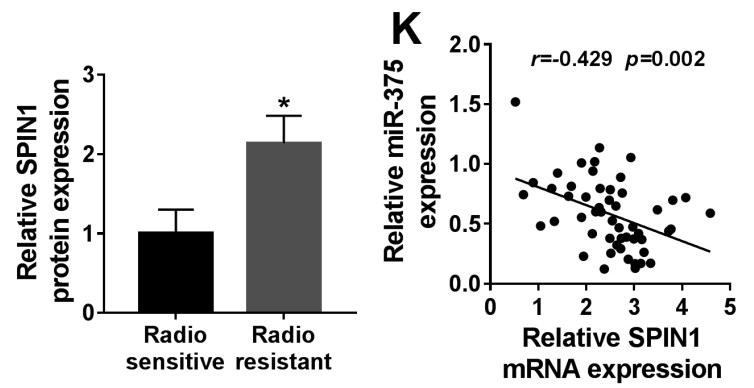

Figure 5. SPIN1 was bound by miR-375. A) The binding site between miR-375 and SPIN1 was analyzed by starBase. B, C) Dual-luciferase reporter assay was conducted to validate the interaction between miR-375 and SPIN1. D, E) The expression of SPIN1 in A549 and H1299 cells transfected with miR375 or anti-miR-375 was detected by western blot. F) The expression of SPIN1 in BEAS-2B, A549, and H1299 cells was detected by western blot. G, H) The expression of SPIN1 in NSCLC tissues $(n=52)$ and normal tissues $(n=52)$ was detected by qRT-PCR and western blot. I, J) The expression of SPIN1 in radioresistant $(n=23)$ or radiosensitive $(n=29)$ NSCLC tissues was detected by qRT-PCR and western blot. K) The correlation between miR-375 and SPIN1 was analyzed by Spearman's correlation analysis. ${ }^{*} \mathrm{p}<0.05$

compared to vector (Figure 6B). In A549 and H1299 cells treated with radiation $(0.2,4$, and $8 \mathrm{~Gy})$, the decreased survival fraction caused by circ_0086720 knockdown was substantially restored by the reintroduction of oe-SPIN1 (Figure 6C). In addition, 4 Gy radiation treatment-induced cell apoptosis was further stimulated in cells transfected with si-circ\#1+vector compared to si-NC+vector but largely suppressed in cells transfected with si-circ\#1+oe-SPIN1 compared to si-circ\#1+vector (Figure 6D). The activities of caspase 3 and caspase 9 were further reinforced in the $4 \mathrm{~Gy}+\mathrm{si}$ circ\#1+vector group compared to the $4 \mathrm{~Gy}$-si-NC+vector group but partially suppressed in the $4 \mathrm{~Gy}+$ si-circ\#1+oeSPIN1 group compared to the $4 \mathrm{~Gy}+$ si-circ\#1+vector group (Figures 6E, 6F). The expression of $\gamma \mathrm{H} 2 \mathrm{AX}$ displayed the 

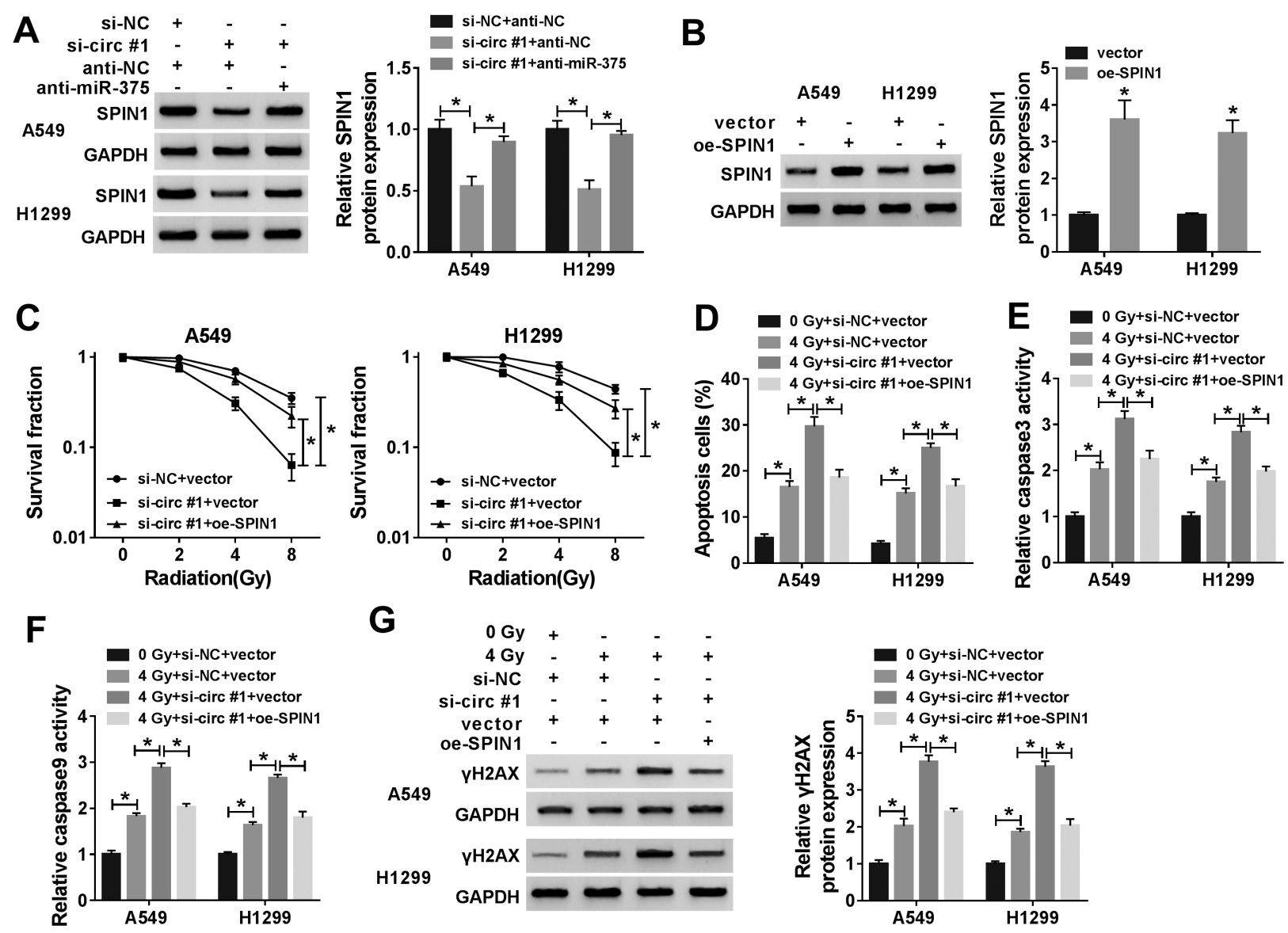

Figure 6. SPIN1 overexpression weakened the enhancement effect of circ_0086720 knockdown on radiosensitivity. A) The expression of SPIN1 in A549 and H1299 cells transfected with si-NC+anti-NC, si-circ\# 1+anti-miR-375, and si-circ\# 1+anti-NC was measured by western blot. B) The expression of SPIN1 in cells transfected with oe-SPIN1 or vector was measured by western blot. A549 and H1299 cells transfected with si-NC+vector, sicirc\#1+vector or si-circ\# 1+oe-SPIN1 and exposed to radiation $(0,2,4$, and $8 \mathrm{~Gy}), \mathrm{C})$ cell survival fraction was detected using colony formation assay. D) Cell apoptosis was checked using a flow cytometry assay. E, F) The activities of caspase 3 and caspase 9 were assessed using the corresponding kits. G) The expression of $\gamma \mathrm{H} 2 \mathrm{AX}$ was examined by western blot. ${ }^{\star} \mathrm{p}<0.05$

same tendency as caspase 3 and caspase 9 activities in A549 and $\mathrm{H} 1299$ cells with different transfection (Figure 6G). These data expressed that circ_0086720 knockdown enhanced the radiosensitivity by lessening SPIN1 expression by mediating miR-375.

Circ_0086720 knockdown increased radiosensitivity in vivo. A549 cells transfected with sh-circ or sh-NC were implanted into nude mice to allow tumor growth. The irradiation (4 Gy) was implemented when tumor volume reached $300 \mathrm{~mm}^{3}$. After radiation, the body weight of mice among three groups had no significant difference, suggesting all mice grew normally (Figure 7A). Tumor volume was significantly reduced compared to that without radiation, and circ_0086720 knockdown further declined tumor volume and bodyweight (Figure 7B). After $28 \mathrm{~d}$, tumor tissues were removed. The representative images of tumors are shown in Figure 7C, and tumor weight in the sh-circ group was notably lower than that in the sh-NC group (Figure 7D). All data showed that circ_0086720 knockdown enhanced radiosensitivity to further block tumor growth.

\section{Discussion}

The biological complexity and heterogeneity of cancers make certain types of tumors often resistant to radiation therapy [25]. Cancer radiation resistance is associated with enhanced local invasion, metastasis, and poor prognosis [26]. Therefore, elucidating the biological characteristics of radiation resistance is essential to ensure the effectiveness of radiation therapy. A large number of previous studies have introduced that long non-coding RNAs (lncRNAs) and miRNAs involved in the radioresistance of human cancers, including cervical cancer, breast cancer, and NSCLC [27-29]. However, few studies demonstrated the function of circRNAs in radioresistance of cancers. Shuai et al. stated that circ_0000285 regulated the radiosensitivity of nasopharyngeal carci- 
A

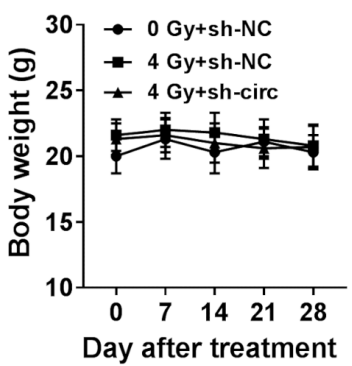

C

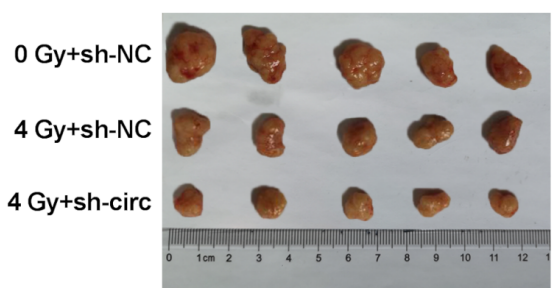

B

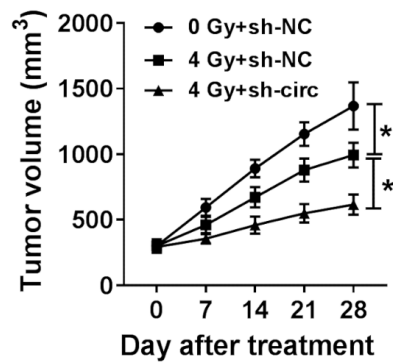

D

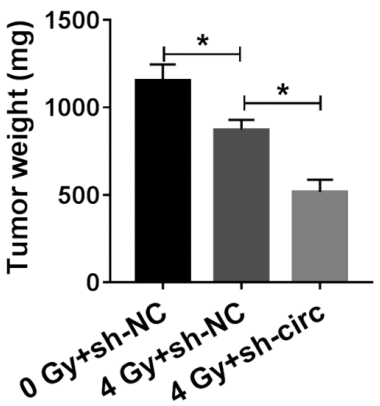

Figure 7. Circ_0086720 knockdown enhanced radiosensitivity in vivo. A549 cells transfected with sh-circ or sh-NC were injected into nude mice followed by the radiation ( 0 or $4 \mathrm{~Gy}$ ). A, B) Body weight of mice and tumor volume were measured once a week after radiation treatment. C) After $28 \mathrm{~d}$, tumor nodes were removed and photographed. D) Tumor weight was measured at $28 \mathrm{~d}$ post-radiation. ${ }^{\star}$ p $<0.05$

noma and served as a prognostic indicator [30]. Wang et al. addressed that circ_0001313 was remarkably upregulated in colon cancer cells treated with radiation, and circ_0001313 silence enhanced radiosensitivity to block cell viability and colony formation [13]. Su et al. utilized circRNA microarray to identify differently expressed circRNAs in radioresistant esophageal cancer cells and parental cells, and circ_0086720 was highly expressed in radioresistant esophageal cancer cells [11], hinting that circ_0086720 might play a vital role in radioresistance of esophageal cancer. However, the exact functions of circ_0086720 were not evaluated in any cancers. In our study, we, for the first time, explored the function of circ_0086720 in NSCLC cells with radiation treatment and found that circ_0086720 knockdown could reinforce radiosensitivity to further impair cell survival and induce cell apoptosis. Moreover, the similar role of circ_0086720 that enhanced radiosensitivity was also identified in vivo, indicating its role in radiotherapy resistance.

CircRNA-miRNA-mRNA network is a widely recognized mode of action in cancer progression [31]. We deduced that circ_0086720 also functioned as a competing endogenous RNA (ceRNA) by targeting downstream miRNAs. Through bioinformatics analysis, a dual-luciferase reporter assay, RIP, and RNA pull-down assay, miR-375 was validated as a target of circ_0086720. miR-375 was reported to be downregulated in NSCLC [32]. Consistently, our study also proved that miR-375 expression was lessened in NSCLC tissues and decreased more in radioresistant NSCLC tissues. Previous studies also introduced that miR-375 could promote the radiosensitivity of cervical cancer cells and oral squamous cell carcinoma by targeting downstream mRNAs [33, 34]. In agreement with these findings, we found that miR-375 downregulation attenuated circ_0086720 knockdowninduced radiosensitivity of NSCLC cells, indicating that miR-375 also mediated radioresistance in NSCLC.

SPIN1 was targeted by miR-375 and aberrantly upregulated in radioresistant NSCLC tissues. Further analysis discovered that the expression of SPIN1 was declined in NSCLC cells with circ_0086720 knockdown, while the reintroduction of miR-375 inhibitor increased SPIN1 expression. A previous study declared that SPIN1 expression was elevated in NSCLC tissues and cells, and overexpression of SPIN1 aggravated NSCLC development, leading to poor prognosis [35]. Besides, the similar role of SPIN1 was also mentioned in various cancers, including breast cancer and glioma, and SPIN1 restoration enhanced adriamycin resistance in breast cancer cells $[23,36]$. Our data for the first time explored the role of SPIN1 in radioresistance and proposed that SPIN1 overexpression enhanced radioresistance suppressed by circ_0086720 knockdown in NSCLC cells, thus promoting cell survival and repressing cell apoptosis.

Collectively, our study mainly disclosed that circ_0086720 knockdown could strengthen the radiosensitivity of NSCLC by targeting the miR-375/SPIN1 pathway. This paper first 
explored the role of circ_0086720 in radioresistance of NSCLC and provided an underlying regulatory mechanism for circ_0086720, implying that circ_0086720 might be used as an indicator of NSCLC radiotherapy.

Acknowledgments: This study was supported by the Project Plan of the Hebei Medical Science Research (Grant No. 20190953).

\section{References}

[1] SIEGEL R, NAISHADHAM D, JEMAL A. Cancer statistics, 2013. CA Cancer J Clin 2013; 63: 11-30. https://doi. org/10.3322/caac. 21166

[2] TAYLOR MD, NAGJI AS, BHAMIDIPATI CM, THEODOSAKIS N, KOZOWER BD et al. Tumor recurrence after complete resection for non-small cell lung cancer. Ann Thorac Surg 2012; 93: 1813-1820; discussion 1820-1811. https:// doi.org/10.1016/j.athoracsur.2012.03.031

[3] SONG L, PENG L, HUA S, LI X, MA L et al. miR-144-5p enhances the radiosensitivity of non-small-cell lung cancer cells via targeting ATF2. Biomed Res Int 2018; 2018: 5109497. https://doi.org/10.1155/2018/5109497

[4] WILlERS H, AZZOLI CG, SANTIVASI WL, XIA F. Basic mechanisms of therapeutic resistance to radiation and chemotherapy in lung cancer. Cancer J 2013; 19: 200-207. https://doi.org/10.1097/PPO.0b013e318292e4e3

[5] BAUMANN M, KRAUSE M, ZIPS D, PETERSEN C, DITTMANN K et al. Molecular targeting in radiotherapy of lung cancer. Lung Cancer 2004; 45 Suppl 2: S187-197. https://doi. org/10.1016/j.lungcan.2004.07.975

[6] LE PECHOUX C. Role of postoperative radiotherapy in resected non-small cell lung cancer: a reassessment based on new data. Oncologist 2011; 16: 672-681. https://doi. org/10.1634/theoncologist.2010-0150

[7] WANG WT, HAN C, SUN YM, CHEN TQ, CHEN YQ. Noncoding RNAs in cancer therapy resistance and targeted drug development. J Hematol Oncol 2019; 12: 55. https://doi. org/10.1186/s13045-019-0748-Z

[8] ZHAO W, DONG M, PAN J, WANG Y, ZHOU J et al. Circular RNAs: A novel target among noncoding RNAs with potential roles in malignant tumors (Review). Mol Med Rep 2019; 20: 3463-3474. https://doi.org/10.3892/mmr.2019.10637

[9] CORTES-LOPEZ M, MIURA P. Emerging Functions of Circular RNAs. Yale J Biol Med 2016; 89: 527-537. PMID: 28018143

[10] DONG WW, LI HM, QING XR, HUANG DH, LI HG. Identification and characterization of human testis derived circular RNAs and their existence in seminal plasma. Sci Rep 2016; 6: 39080. https://doi.org/10.1038/srep39080

[11] SU H, LIN F, DENG X, SHEN L, FANG Y et al. Profiling and bioinformatics analyses reveal differential circular RNA expression in radioresistant esophageal cancer cells. J Transl Med 2016; 14: 225. https://doi.org/10.1186/s12967-016-0977-7

[12] YU D, LI Y, MING Z, WANG H, DONG Z et al. Comprehensive circular RNA expression profile in radiation-treated HeLa cells and analysis of radioresistance-related circRNAs. PeerJ 2018; 6: e5011. https://doi.org/10.7717/peerj.5011
[13] WANG L, PENG X, LU X, WEI Q, CHEN M et al. Inhibition of hsa_circ_0001313 (circCCDC66) induction enhances the radio-sensitivity of colon cancer cells via tumor suppressor miR-338-3p: Effects of cicr_0001313 on colon cancer radiosensitivity. Pathol Res Pract 2019; 215: 689-696. https://doi. org/10.1016/j.prp.2018.12.032

[14] LIU J, XUE N, GUO Y, NIU K, GAO L et al. CircRNA_100367 regulated the radiation sensitivity of esophageal squamous cell carcinomas through miR-217/Wnt3 pathway. Aging (Albany NY) 2019; 11: 12412-12427. https://doi.org/10.18632/ aging. 102580

[15] CHEN L, ZHOU H, GUAN Z. CircRNA_000543 knockdown sensitizes nasopharyngeal carcinoma to irradiation by targeting miR-9/platelet-derived growth factor receptor B axis. Biochem Biophys Res Commun 2019; 512: 786-792. https://doi.org/10.1016/j.bbrc.2019.03.126

[16] WANG L, ZHENG C, WU X, ZHANG Y, YAN S et al. CircSOX4 promotes non-small cell lung cancer progression by activating the Wnt/beta-catenin pathway. Mol Oncol 2020. https://doi.org/10.1002/1878-0261.12656

[17] XU Z, XIANG W, CHEN W, SUN Y, QIN F et al. Circ-IGF1R inhibits cell invasion and migration in non-small cell lung cancer. Thorac Cancer 2020. https://doi.org/10.1111/17597714.13329

[18] MACFARLANE LA, MURPHY PR. MicroRNA: Biogenesis, function and role in cancer. Curr Genomics 2010; 11: 537561. https://doi.org/10.2174/138920210793175895

[19] LU J, ZHAN Y, FENG J, LUO J, FAN S. MicroRNAs associated with therapy of non-small cell lung cancer. Int J Biol Sci 2018; 14: 390-397. https://doi.org/10.7150/ijbs.22243

[20] LI Y, JIANG Q, XIA N, YANG H, HU C. Decreased expression of microRNA-375 in nonsmall cell lung cancer and its clinical significance. J Int Med Res 2012; 40: 1662-1669. https://doi.org/10.1177/030006051204000505

[21] ZHANG P, CONG B, YUAN H, CHEN L, LV Y et al. Overexpression of spindlin1 induces metaphase arrest and chromosomal instability. J Cell Physiol 2008; 217: 400-408. https:// doi.org/10.1002/jcp.21515

[22] FANG Z, CAO B, LIAO JM, DENG J, PLUMMER KD et al. SPIN1 promotes tumorigenesis by blocking the uL18 (universal large ribosomal subunit protein 18)-MDM2-p53 pathway in human cancer. Elife 2018; 7. https://doi.org/10.7554/ eLife.31275

[23] CHEN X, WANG YW, GAO P. SPIN1, negatively regulated by miR-148/152, enhances Adriamycin resistance via upregulating drug metabolizing enzymes and transporter in breast cancer. J Exp Clin Cancer Res 2018; 37: 100. https:// doi.org/10.1186/s13046-018-0748-9

[24] MA W, MA CN, ZHOU NN, LI XD, ZHANG YJ. Up- regulation of miR-328-3p sensitizes non-small cell lung cancer to radiotherapy. Sci Rep 2016; 6: 31651. https://doi.org/10.1038/ srep31651

[25] CHI HC, TSAI CY, TSAI MM, YEH CT, LIN KH. Roles of long noncoding RNAs in recurrence and metastasis of radiotherapy-resistant cancer stem cells. Int J Mol Sci 2017; 18. https://doi.org/10.3390/ijms18091903 
[26] LEE SY, JEONG EK, JU MK, JEON HM, KIM MY et al. Induction of metastasis, cancer stem cell phenotype, and oncogenic metabolism in cancer cells by ionizing radiation. Mol Cancer 2017; 16: 10. https://doi.org/10.1186/s12943-0160577-4

[27] FAN L, HUANG C, LI J, GAO T, LIN Z et al. Long noncoding RNA urothelial cancer associated 1 regulates radioresistance via the hexokinase $2 /$ glycolytic pathway in cervical cancer. Int J Mol Med 2018; 42: 2247-2259. https://doi.org/10.3892/ ijmm. 2018.3778

[28] WANG B, ZHENG J, LI R, TIAN Y, LIN J et al. Long noncoding RNA LINC02582 acts downstream of miR-200c to promote radioresistance through $\mathrm{CHK} 1$ in breast cancer cells. Cell Death Dis 2019; 10: 764. https://doi.org/10.1038/ s41419-019-1996-0

[29] LIU AM, ZHU Y, HUANG ZW, LEI L, FU SZ et al. Long noncoding RNA FAM201A involves in radioresistance of non-small-cell lung cancer by enhancing EGFR expression via miR-370. Eur Rev Med Pharmacol Sci 2019; 23: 58025814. https://doi.org/10.26355/eurrev_201907_18319

[30] SHUAI M, HONG J, HUANG D, ZHANG X, TIAN Y. Upregulation of circRNA_0000285 serves as a prognostic biomarker for nasopharyngeal carcinoma and is involved in radiosensitivity. Oncol Lett 2018; 16: 6495-6501. https://doi. org/10.3892/ol.2018.9471

[31] JIA N, TONG H, ZHANG Y, KATAYAMA H, WANG Y et al. CeRNA expression profiling identifies KIT-Related circRNA-miRNA-mRNA networks in gastrointestinal stromal tumour. Front Genet 2019; 10: 825. https://doi.org/10.3389/ fgene.2019.00825
[32] KUMAR S, SHARAWAT SK, ALI A, GAUR V, MALIK PS et al. Identification of differentially expressed circulating serum microRNA for the diagnosis and prognosis of Indian non-small cell lung cancer patients. Curr Probl Cancer 2020: 100540. https://doi.org/10.1016/j.currproblcancer.2020.100540

[33] SONG L, LIU S, ZENG S, ZHANG L, LI X. miR-375 Modulates Radiosensitivity of HR-HPV-Positive Cervical Cancer Cells by Targeting UBE3A through the p53 Pathway. Med Sci Monit 2015; 21: 2210-2217. https://doi.org/10.12659/ MSM.893859

[34] ZHANG B, LI Y, HOU D, SHI Q, YANG S et al. MicroRNA-375 Inhibits Growth and Enhances Radiosensitivity in Oral Squamous Cell Carcinoma by Targeting Insulin Like Growth Factor 1 Receptor. Cell Physiol Biochem 2017; 42: 2105-2117. https://doi.org/10.1159/000479913

[35] SONG Q, JI Q, XIAO J, LI F, WANG L et al. miR-409 Inhibits Human Non-Small-Cell Lung Cancer Progression by Directly Targeting SPIN1. Mol Ther Nucleic Acids 2018; 13: 154-163. https://doi.org/10.1016/j.omtn.2018.08.020

[36] LI Y, MA X, WANG Y, LI G. miR-489 inhibits proliferation, cell cycle progression and induces apoptosis of glioma cells via targeting SPIN1-mediated PI3K/AKT pathway. Biomed Pharmacother 2017; 93: 435-443. https://doi.org/10.1016/j. biopha.2017.06.058 